\title{
Effects of Porcine Placenta Extract Ingestion on Ultraviolet B-induced Skin Damage in Hairless Mice
}

\author{
Ki-Bae Hong ${ }^{1}$, Yooheon Park ${ }^{2}$, Jae Hwan Kim³ ${ }^{3}$ Jin Man Kim, and Hyung Joo Suh ${ }^{1,2, *}$ \\ ${ }^{1}$ Department of Public Health Science, Graduate School, Korea University, Seoul 136-713, Korea \\ ${ }^{2}$ Department of Food and Nutrition, Korea University, Seoul 136-713, Korea \\ ${ }^{3}$ Neo Cremar Co. Ltd., Sungnam 462-806, Korea \\ ${ }^{4}$ Department of Food Science and Biotechnology of Animal Resources, Konkuk University, Seoul 143-701, Korea
}

\begin{abstract}
The aim of our study was to evaluate the potential benefits of an oral supplement containing porcine placenta extract (PPE) on skin parameters related to cutaneous physiology and aging. PPEs were administered orally to hairless mice for $12 \mathrm{wk}$. The effects of oral PPE administration on skin water-holding capacity and Transepidermal Water Loss (TEWL) were similar to those of oral collagen (HYCPU2) administered as a positive control. Magnified photographs and replica images showed a reduction in UVB-induced wrinkle formation after collagen and PPE treatments. PPE treatments ameliorated the thicker skin surface that results from UVB exposure, based on a histological examination of skin tissue. The groups that were orally administered PPE $(0.05 \%, \mathrm{OL} ; 0.1 \%$, OH group) showed significantly reduced Matrix Metaloproteinase-2 (MMP-2) mRNA expression levels compared with the UVB control (Con), by $33.5 \%$ and $35.2 \%$, respectively. The mRNA expression of another collagen-degrading protein, MMP-9, was also significantly lower in the groups that received oral administration of PPE (especially in the OH group) than in the control group. Additionally, oral administration of PPE significantly upregulated tissue inhibitor of metalloproteinase-1 (TIMP-1) and -2 mRNA expression levels compared with expression levels in the control group $(p<0.05)$. This indicates that orally administered PPE activated the expression of Timp-1 and -2 , inhibitors of MMP, which is responsible for collagen degradation in skin. Taken together, we propose that long-term oral administration of PPE might have a beneficial effect with respect to skin photo-aging.
\end{abstract}

Keywords: porcine placenta extract, photoprotective activity, water holding capacity, skin damage

Received April 30, 2015; Revised May 25, 2015; Accepted May 26, 2015

\section{Introduction}

In recent years, functional foods claiming health benefits have increased remarkably. The relationship between food and skin has drawn considerable attention due to the physiological effects of some dietary compounds on the skin aging process. Corresponding to this trend, an increasing number of products claim to aid this pursuit ( $\mathrm{Za}-$ gue, 2008).

An important trend in skin care is the use of diet and oral supplements to improve the skin's appearance and structure. Healthy skin reflects general health status and accordingly the skin is influenced by the consumption of dietary substances, including vitamins, antioxidants, fatty

\footnotetext{
*Corresponding author: Hyung Joo Suh, Department of Public Health Science, Graduate School, Korea University, Seoul 136713, Korea. Tel: +82-2-3290-5639, Fax: +82-2-940-2859, E-mail: suh1960@korea.ac.kr
}

acids, and hydrolyzed proteins (Draelos, 2010). Therefore, the effects of nutritional factors on the skin have received increasing attention, and a number of clinical studies have indicated that dietary supplementation can modulate skin functions (Zague, 2008). Cosgrove et al. (2007) evaluated the associations between nutrient intake and skin aging parameters (wrinkled appearance, senile dryness, and skin atrophy) and concluded that higher vitamin $\mathrm{C}$ and linoleic acid intake and lower fat and carbohydrate intake are associated with better skin appearance.

Placenta extract (PE) is widely used in the clinical and cosmetic fields, and possesses marked antioxidant activity (Togashi et al., 2002). The placenta is as a natural storehouse of biologically active compounds including hormones, proteins, peptides, growth factors, nucleic acids, glycosaminoglycans, and polydeoxyribonucleotides crucial for fetal growth and development (Zague, 2008). PE contains enzymes such as alkaline acid phosphatase and glutamic oxaloacetic transaminase, as well as nucleic 
acids, vitamins, amino acids, steroids, fatty acids, and minerals (Ansari et al., 1994). It is a traditional medicine used for wound healing owing to its immunotropic and anti-inflammatory properties, and is used as a cosmetic supplement in many Asian countries (Tonello et al., 1996).

Various placental products are manufactured in various countries. Many are used for therapeutic purposes and the remainder are mainly used as skin creams and lotions (Chakraborty et al., 2009). The therapeutic products are mostly injectable, although a few can be taken orally. Recently, porcine PE (PPE) was developed as an oral supplement (Koike et al., 2012; Wu et al., 2003). Because PPE is relatively safe and its effect is similar to that of human PE (HPE), it is widely used as a cosmetic supplement. PPE has great potential for the treatment of skin wrinkles. No abnormal clinical signs or toxicity of swine placenta extract were detected and placenta extract frequently used as a composition of ointments for skin (Mitsui et al., 2015). However, its anti-wrinkle effects and mechanism of action have not yet been examined in detail. Furthermore, its effects on ultraviolet B (UVB) irradiation-induced skin damage have not been investigated.

Dietary consumption of food supplements has been found to modulate skin functions and can therefore be useful in the treatment of skin aging. Although there is convincing preclinical evidence that PPE ingestion improves skin conditions and even protects skin from UV damage, little is known about the clinical effects of PPE on skin parameters and health. The aim of our study was to evaluate the potential benefits of an oral supplement containing PPE on skin parameters related to cutaneous physiology and aging, including skin elasticity, hydration, roughness, and transepidermal water loss (TEWL).

\section{Materials and Methods}

\section{Preparation of PPE}

PPE was obtained from Neo Cremar Co. Ltd. (Korea). In brief, porcine placenta obtained after normal delivery was washed thoroughly with water. The washed placenta was minced, and then hydrolyzed with protease at neutral $\mathrm{pH}$. The protease which had been used for hydrolysis was inactivated by elevating the temperature of the solution to $90^{\circ} \mathrm{C}$. Insoluble materials remained in the placental solution after hydrolysis was removed by filtration at acidic $\mathrm{pH}$. The filtrate was concentrated with evaporator, and the resulting concentrate was sterilized by heating the temperature of the solution up to $90^{\circ} \mathrm{C}$. Finally, PPE powder was prepared by spray-drying of the concentrated pla- cental extract solution and used for the experiments.

\section{Animals and treatment}

The experimental protocol was reviewed and approved by the Korea University Animal Care Committee (KUIACUC-20130430-2). Male SKH-1 hairless mice were obtained at 6 wk of age $(24 \pm 1 \mathrm{~g})$ from commercial stock (Central Laboratory Animal Inc., Korea). The mice were individually housed in plastic cages with grated stainless steel covers. The room was maintained at $23 \pm 1^{\circ} \mathrm{C}$ with 60 $\pm 5 \%$ atmospheric humidity on a 12 -h light/12-h dark cycle. The mice had ad libitum access to water and a commercial diet (Samyang Co., Korea). After a 1-wk acclimation period, the mice were randomly divided into 5 groups $(6$ mice/group): (1) Normal group (NC; untreated water intake without UBV irradiation), (2) UV control group (Con; untreated water intake with UVB irradiation), (3) positive control [PO; 0.1\% hydrolyzed collagen (HACP-U2, Jellice Co., Sendai, Japan) in drinking water with UVB irradiation], (4) $0.05 \% \mathrm{PPE}$ administration group (OL; 0.05\% PPE in drinking water with UVB irradiation), and (5) $0.1 \%$ PPE administration group $(\mathrm{OH} ; 0.1 \%$ PPE in drinking water with UVB). Treatment was carried out for 12 wk. For oral administration, PPE was administered via drinking water once daily $(0.05 \%$ and $0.1 \%$ PPE in drinking water, equivalent to a dose of 0.1 and $0.2 \mathrm{~g} / \mathrm{kg}$ body weight, respectively, based on daily water consumption).

UVB irradiation was performed according to a previously described method (Gueniche et al., 2006). Briefly, dorsal skin was exposed to UVB light (FLB20SBL, Sankyo Denki, Japan). This source emits light in the wavelength range of 270-400 $\mathrm{nm}$ with an output peak at 313 $\mathrm{nm}$. The hairless mice were exposed to UVB irradiation for four consecutive days with a total daily dose of UVB set at $84 \mathrm{~mJ} / \mathrm{cm}^{2}$ measured by a UV 1700 Radiometer (International Light Inc., Shimadzu, Japan). The animals were irradiated within their cages. Changes in skin properties and gene expression were measured $24 \mathrm{~h}$ later.

\section{Histopathological study}

After 12 wk, animals were sacrificed using sodium pentobarbital. The portion of cutaneous tissue exposed to UVB was quickly removed and fixed in $10 \%$ buffered formalin, embedded in paraffin, and sectioned at $6 \mu \mathrm{m}$. Sections were stained with hematoxylin and eosin dyes (H\&E staining). Histopathological examination was performed. The skin thickness were illuminated under the light (flexible light guide 1500, CarlZeiss, Germany) at an angle of $20^{\circ}$ and analyzed under a stereoscopic micro- 
scope at 7X magnification (Axio Zoom. V16, CarZeiss, Germany) analysis program.

\section{Measurement of skin hydration, transepidermal water loss, and erythema level}

Skin water content was measured using a Corneometer CM 825 with the multi-probe adapter MPA 5 (Courage and Khazaka Electronics GmbH, Germany). The capacitance method, which utilizes the relatively high dielectric constant of water relative to other substances in the skin, was used to measure skin hydration. The measuring probe was pressed onto the skin for $1 \mathrm{~s}$. A change in capacitance is converted into a digital value (arbitrary units), and this value is proportional to the hydration level of the skin. Measurement errors due to skin deformations or evaporation build up can be excluded on the basis of the short measurement time.

TEWL was measured using the Tewameter TM 300 (Courage and Khazaka Electronic $\mathrm{GmbH}$ ) according to the manufacturer's guidelines. The relative humidity was maintained at $40-60 \%$ and the room temperature at 20 $25^{\circ} \mathrm{C}$ during the measurements.

A Mexameter MX 18 (Courage and Khazaka Electronic $\mathrm{GmbH}$ ) was used to quantify the change in erythema levels. Erythema was measured at $660 \mathrm{~nm}$ (the spectral absorption peak of hemoglobin) and $568 \mathrm{~nm}$. The values are indicated as an index (erythema). The accuracy for estimating the erythema contents was $\pm 5 \%$.

\section{Quantitation of mRNA level}

Total RNA was isolated from skin samples using Trizol ${ }^{\circledR}$ Reagent (Invitrogen, USA) according to the manufacturer's protocol. Complementary DNA was synthesized by reverse transcription of $1 \mu \mathrm{g}$ of total RNA using the RevertAid $^{\mathrm{TM}}$ First Strand cDNA Synthesis Kit (Thermo Scientific Fisher, Waltham, USA). Each sample was treated with RQ1 RNase-free DNase I (Promega, USA) according to the manufacturer's protocol. The data were analyzed using the comparative $\mathrm{C}_{\mathrm{T}}\left(2^{-\Delta \Delta \mathrm{CT}}\right)$ method (Schmittgen and Livak, 2008). Quantitative real-time PCR (StepOne Plus Real-Time PCR System, Applied Biosystems, USA) was conducted with TaqMan gene expression assays (Applied Biosystems, USA) and normalized to the endogenous control glyceraldehyde 3-phosphate dehydrogenase.

\section{Statistical analyses}

All statistical analyses were performed using Statistical Package for Social Sciences version 12.0 (SPSS, USA).
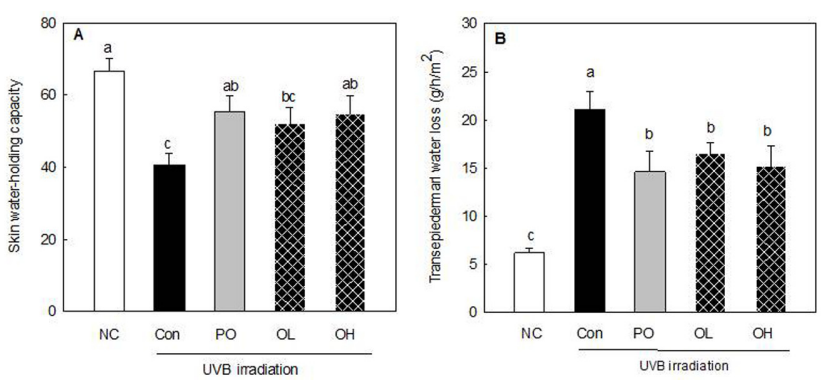

Fig. 1. Effect of PPE on skin water-holding capacity (A) and transepidermal water loss (B) in UVB-irradiated hairless mice. NC: Drinking water intake without UVB irradiation; Con: Drinking water intake with UVB irradiation; PO: $0.1 \%$ hydrolyzed collagen (HACP-U2) intake in drinking water with UVB irradiation; OL: $0.05 \%$ PPE intake in drinking water with UVB irradiation; $\mathrm{OH}: 0.1 \%$ PPE intake in drinking water with UVB. Values are mean \pm SEM ( $\mathrm{n}=6)$. Means with different letters indicate significant differences at $p<0.05$.

All data are reported as means \pm standard error of the mean (SEM). Significant differences in all parameters between the UVB irradiation control group (Con) and the other groups were analyzed using $t$-tests.

\section{Results}

\section{Physical characteristics of the skin}

The water-holding capacity and TEWL of the skin samples are summarized in Fig. 1. UVB irradiation significantly reduced the skin water-holding capacity of the mice (NC $66.52 \%$ vs. Con $40.60 \%, p<0.05$ ). The water-holding capacity of the UVB-irradiated skin was significantly higher in the groups that received oral administration of PO and PPE (OH groups) than in the control mice ( $p<$ $0.05)$. Figure 1B presents the effects of PPE treatment on TEWL in the UVB-irradiated mice. TEWL was significantly higher for mice treated with UVB irradiation than for control mice (NC $6.19 \mathrm{~g} / \mathrm{h} / \mathrm{m}^{2}$ vs. Con $21.09 \mathrm{~g} / \mathrm{h} / \mathrm{m}^{2}, p$ $<0.01)$. When the mice were administered PPE and PO, the UVB-induced increase in TEWL relative to the UVB control was significantly suppressed $(p<0.05)$. The effects of oral PPE administration on the skin water-holding capacity and TEWL were similar to those of hydrolyzed collagen administration, which was considered a positive control.

\section{Erythema and melanin values}

The effects of PPE treatment on erythema and melanin values in the UVB-irradiated hairless mice are shown in Fig. 2. Erythema values were significantly higher for UVB- 

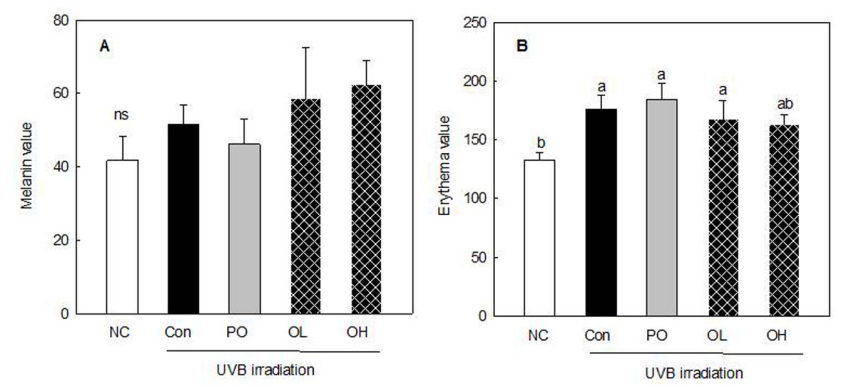

Fig. 2. Effect of PPE on melanin (A) and erythema values (B) in UVB-irradiated hairless mice. NC: Drinking water intake without UVB irradiation; Con: Drinking water intake with UVB irradiation; PO: $0.1 \%$ hydrolyzed collagen (HACP-U2) intake in drinking water with UVB irradiation; OL: $0.05 \%$ PPE intake in drinking water with UVB irradiation; $\mathrm{OH}: 0.1 \%$ PPE intake in drinking water with UVB. Values are mean \pm SEM $(n=6)$. Means with different letters indicate significant differences at $p<0.05$.

irradiated mice than control mice (NC 132.46 vs. Con 176.24, $p<0.05)$. PO and PPE treatments did not suppress the UVB-induced increase in erythema values. However, PPE treatment showed a tendency toward decreased erythema values. UVB irradiation did not significantly increase melanin values in the mice. Furthermore, PO and PPE treatments did not suppress the UVB-induced increase in melanin values. Erythema value also was not significantly influence by the treatments (Fig. 2B).

\section{Wrinkle formation and histopathology of the skin}

Fig. 3 shows photographs and replica images of the dorsal skin of the UVB-irradiated hairless mice treated with PPE. Male hairless mice chronically exposed to UVB radiation showed deep, irregular wrinkles on the dorsal skin. Wrinkle formation in the UVB-control group (Con) was greater than that in the Normal group (NC). Wrinkle formation due to intrinsic aging was also observed in non-UVB-irradiated hairless mice. The magnified photographs and replica images show a reduction in UVBinduced wrinkle formation after PO and PPE treatments. No significant difference in wrinkle formation in the UVB-irradiated dorsal skin was observed between the PO and PPE treatment groups.

To evaluate the histological status of skin tissue after PPE treatment, H\&E staining was performed on dorsal skin tissue samples (Fig. 4A). UVB treatment resulted in a hyperplasia phenotype based on H\&E staining of skin tissue; this presents a thicker epidermal surface than that of mice in the control group (Fig. 4). This result indicates that UVB induced photo-ageing on the skin surface. PPE


B

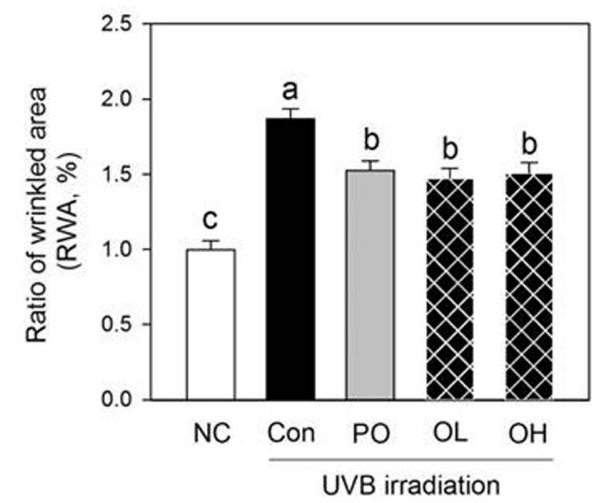

Fig. 3. Effect of PPE on wrinkle formation (A) and ratio of wrinkled area (B) of dorsal skin in hairless mice. $\mathrm{NC}$ : Drinking water intake without UVB irradiation; Con: Drinking water intake with UVB irradiation; PO: $0.1 \%$ hydrolyzed collagen (HACP-U2) intake in drinking water with UVB irradiation; OL: $0.05 \%$ PPE intake in drinking water with UVB irradiation; $\mathrm{OH}$ : $0.1 \%$ PPE intake in drinking water with UVB. Values are mean $\pm \operatorname{SEM}(n=6)$. Means with different letters indicate significant differences at $p<0.05$.

treatments ameliorate the thicker skin surface caused by UVB exposure. Compared with the control group, the groups that received oral PPE treatment showed a significant decrease in epidermal thickness $(p<0.05)$, but thickness did not differ significantly from that of the UVB treatment groups. These histological data indicate that PPE treatment protects skin from UVB-induced hyperplasia, consistent with the inhibition of wrinkle formation.

\section{MMPs and TIMPs expression}

The results of the experiments described above indicated that PPE treatment results in the inhibition of UVBinduced wrinkle formation, skin thickening, and water loss. Wrinkle formation and skin dehydration are related to a lack of collagen, an important structural skin component. Therefore, we examined the effect of PPE on the expression levels of genes related to collagen synthesis and degradation. 


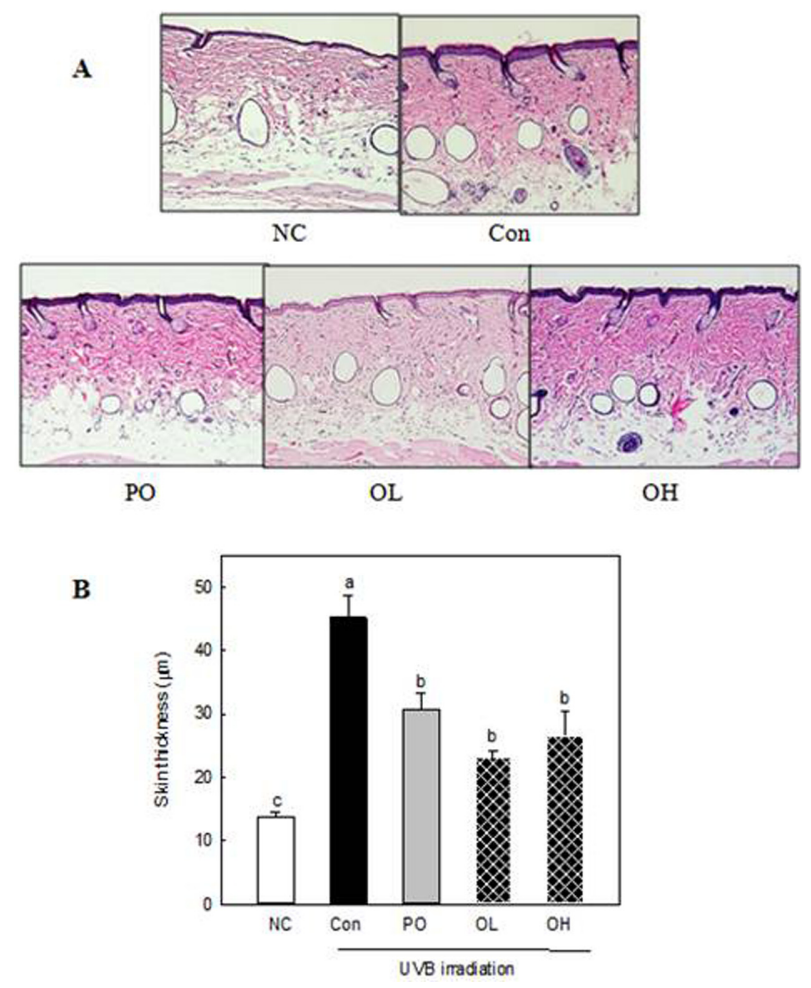

Fig. 4. Epidermal thickness of hairless mice treated with PPE. NC: Drinking water intake without UVB irradiation; Con: Drinking water intake with UVB irradiation; PO: $0.1 \%$ Hydrolyzed collagen (HACP-U2) intake in drinking water with UVB irradiation; OL: $0.05 \%$ PPE intake in drinking water with UVB irradiation; $\mathrm{OH}: 0.1 \%$ PPE intake in drinking water with UVB. Values are mean \pm SEM $(\mathrm{n}=6)$. Means with different letters indicate significant differences at $p<0.05$.

MMP-2 and -9 play important roles in the degradation of collagen in tissues. Significantly lower MMP-2 mRNA expression was observed in groups with oral administration of PPE (OL and $\mathrm{OH}$ groups) than in the control group (Fig. 5A). There was no significant difference in MMP-2 expression between the $\mathrm{OL}$ and $\mathrm{OH}$ groups.

The mRNA expression of the gene encoding another collagen-degrading protein, MMP-9, was also significantly lower in the groups that received oral administration of PPE (especially the $\mathrm{OH}$ group) than in the control group (Fig. 5B). The 0.1\% PPE administration group (OH) exhibited down-regulated MMP-9 expression. However, there were no significant differences in MMP-9 expression between the treated group (OL) and the control group.

Since the regulation of matrix metalloproteinases is coordinated by tissue inhibitors, including TIMP-1 and 2, we investigated whether TIMP-1 and -2 mRNA expression levels were altered in UVB-treated mice. Groups that were orally administered PPE showed significantly up-
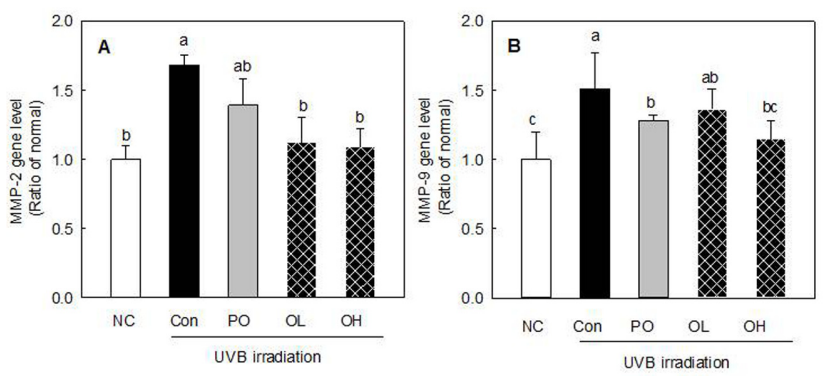

Fig. 5. Effect of PPE on MMP-2 (A) and -9 (B) expression in hairless mice. NC: Drinking water intake without UVB irradiation; Con: Drinking water intake with UVB irradiation; PO: $0.1 \%$ hydrolyzed collagen (HACP-U2) intake in drinking water with UVB irradiation; OL: $0.05 \%$ PPE intake in drinking water with UVB irradiation; $\mathrm{OH}: 0.1 \%$ PPE intake in drinking water with UVB. Values are mean \pm SEM $(\mathrm{n}=6)$. Means with different letters indicate significant differences at $p<0.05$.
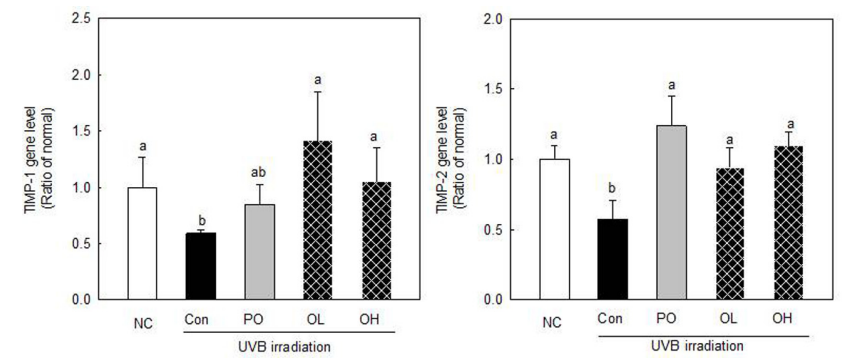

Fig. 6. Effect of PPE on TIMP-1 and TIMP-2 expression in hairless mouse. NC: Drinking water intake without UVB irradiation; Con: Drinking water intake with UVB irradiation; PO: $0.1 \%$ hydrolyzed collagen (HACP-U2) intake in drinking water with UVB irradiation; OL: $0.05 \% \mathrm{PPE}$ intake in drinking water with UVB irradiation; $\mathrm{OH}: 0.1 \%$ PPE intake in drinking water with UVB. Values are mean \pm SEM $(\mathrm{n}=6)$. Means with different letters indicate significant differences at $p<0.05$.

regulated TIMP-1 and -2 mRNA expression levels compared with those in the control group $(p<0.05)$. There were no significant differences in TIMP expression between the $\mathrm{OL}$ and $\mathrm{OH}$ treatment groups. This indicates that orally administered PPE activated the expression of TIMP-1 and -2, inhibitors of MMPs, which is responsible for collagen degradation in the skin.

\section{Discussion}

PPE is likely to contain many specific and potent bioactive substances from a nutritional perspective given their competitive living conditions (Koike et al., 2012). Owing to its various bioactive properties, PPE has gained inc- 
reasing popularity as an ingredient in functional foods and pharmaceuticals (Han et al., 2013). To the best of our knowledge, this is the first study revealing the effects of long-term oral administration of PPE on UVB-induced skin aging in vivo. Moreover, the effects of PPE and the mechanism underlying increased water holding and antiwrinkle capacity were elucidated.

Effects of PE on the regulation of biological responses and its potential as a therapeutic reagent for various diseases have been implicated in numerous studies. Nevertheless, solid evidence demonstrating the biological mechanisms of these effects in a well-established experimental system is still lacking. In this study, we demonstrate that the administration of PE increases the waterholding capacity and anti-wrinkle effect in UVB-induced hairless mice.

The ability of the skin to hold water is related to the lipid content within the stratum corneum, which plays a predominant role in maintaining the skin barrier function (Madison, 2003), and also to dermal glycosaminoglycans, which have a high water-holding capacity (Oh et al., 2011). In our study, oral PPE administration improved UVBinduced hydration changes, the skin water-holding capacity, and TEWL in the UVB-irradiated hairless mice (Fig. 1).

Placenta preparation involves several processes including freeze-drying, boiling, and hydrolysis. An aqueous extract of placenta contains amino acids, peptides, glycosaminoglycans, lipids, polynucleotides (mainly polydeoxyribonucleotide fragments), vitamins, minerals, etc. Thus, biologically active proteins or other large molecules in the placenta can be degraded or inactivated, and small molecules, such as estrogen, progesterone, and growth factors, may remain as active components (Chakraborty and Bhattacharyya, 2012). Of the substances used in cosmetic products, freeze-dried PE seems to be the most effective in strengthening flaccid connective tissue (Bohn, 1985). PE improves the skin's blood circulation and metabolism, and increases the frequency of cell division in the skin (Mittal, 1977). It is a rich source of bioactive peptides and amino acids. Bioactive components in PPE might affect the UVB-induced decrease in water holding capacity and increase in TEWL.

Furthermore, HPE is clinically and cosmetically used for wrinkles because it promotes skin regeneration ( $\mathrm{Pal}$ et al., 1995). HPE is also a component of various skin ointments owing to its effects related to skin revitalization and melanocyte growth, and its pigment-inducing activities; it is also used to treat skin hypersensitivity-like dermatitis and psoriasis (Pal et al., 2002). Oral intake of PPE also showed an anti-wrinkle effect in UVB-induced hairless mice (Fig. 3 and 4). Wrinkles are caused by aging, genetic background, and environmental factors such as UVB irradiation. PPE was recently developed as an oral supplement for the same purposes as HPE, i.e., skin regeneration, and is now clinically used as an anti-wrinkle treatment (Pal et al., 2002; Pal et al., 1995). Yoshikawa et al. (Yoshikawa et al., 2013) demonstrated that oral PPE treatment may be used to improve skin wrinkles. Oral PPE treatment in postmenopausal women leads to a decrease in the width of wrinkles. In our study, oral PPE treatment reduced UVB-induced wrinkle formation (Fig. 3). Skin fibroblasts express extracellular matrix proteins including collagen and play a critical role in skin regeneration. Collagen precursor molecules (procollagen) are synthesized in fibroblasts (Pinnell, 1982). PPE increases fibroblast proliferation (Pal et al., 2002). A recent study has shown that PPE drives skin fibroblasts into the $\mathrm{S}$ phase and increases the basic fibroblast growth factor level (Pal et al., 2002). It significantly increases collagen production in dermal fibroblasts, suggesting that it has a stimulatory effect on collagen production (Yoshikawa et al., 2013). Skin collagen has a central role in the mechanical strength of skin, as a component of the extracellular matrix. A decline in the dermal collagen content is a major factor in wrinkle formation (Calleja-Agius et al., 2007). The reduction in wrinkles caused by PPE treatment may be related to its effects on the skin water-holding capacity and TEWL; PPE may have an anti-wrinkle effect by preventing moisture loss.

UV exposure is a leading cause of elevated MMP expression. High levels of MMPs are known to cause damage to skin connective tissue via collagen degradation and collagen synthesis reduction (Kong et al., 2010). Type IV collagenases, MMP-2 and MMP-9, are UV-inducible MMPs. MMP-9 plays an important role in apoptosis and the fibrillar collagens after initial cleavage by collagenase (Anggakusuma et al., 2010; Onoue et al., 2003). Therefore, the inhibitory effect of PPE against UVB damage was further confirmed based on its ability to block MMP2 and MMP-9 expression. UVB-exposure also affects the expression of genes encoding TIMPs (Onoue et al., 2003). TIMP-1 and TIMP-2 are the major endogenous inhibitors, and regulate the activity of MMP-2 and MMP-9 (Corbel et al., 2000; Kong et al., 2010). The presence of PPE essentially inhibited the UVB effect by suppressing the expression of MMPs and inhibiting the decreased expression of TIMPs genes.

The long-term administration of marine collagen hydro- 
lysate $(\mathrm{MCH})$ from Chum Salmon skin at a $4.5 \%$ concentration can also significantly inhibit the expression of MMP-1 and increase TIMP-1 expression in chronologically aged skin. Therefore, the increased MMP-1 protein and reduced TIMP-1 protein levels together contributed to the inhibitory effect of $\mathrm{MCH}$ on the degradation of collagen fibers in aged skin (Liang et al., 2010).

Several clinical studies have indicated a broader range of skin-related benefits of orally ingested antioxidants. The daily oral ingestion of a mixture of antioxidants containing lutein $(6 \mathrm{mg})$ and zeaxanthin $(0.3 \mathrm{mg})$ induces an increase in skin surface lipids and skin hydration, while simultaneously reducing the amount of oxidized skin lipids following UV light exposure (lipid peroxidation) (Morganti et al., 2002). The delivery rate of orally administered PPE to the skin surface influences its effects. Orally administered PPE might be delivered to cells at the dermal-epidermal junction via the bloodstream or via the sebaceous gland route (Darvin et al., 2006; Palombo et al., 2007). We hypothesized that oral intake of PPE might show greater effects than topical application. Sebaceous delivery could result in greater amounts of PPE at or near the skin surface than might be achieved from a topical route.

We have a limitation in this study is that we have not identified the active proteinous component from PPE. Further investigation is required to identify the actual active component from enzyme treated PPE. The active components will be small molecular compounds since the active component from such products are usually small peptides (tripeptides and tetrapeptides).

In this study, long-term oral ingestion of PPE had beneficial effects on age-related phenomena, including effects on water holding capacity, trans-epidermal water, wrinkles, as well as MMP and TIMP-1 expression. Based on these results, we inferred that long-term oral administration of PPE might be an effective method to slow skin photoaging.

\section{Acknowledgements}

This research was supported by Technology Development Program for Food, Ministry for Food, Agriculture, Forestry and Fisheries, Republic of Korea.

\section{References}

1. Anggakusuma, Yanti, and Hwang, J. K. (2010) Effects of macelignan isolated from Myristica fragrans Houtt. on UVB- induced matrix metalloproteinase- 9 and cyclooxygenase- 2 in HaCaT cells. J. Dermatol. Sci. 57, 114-122.

2. Ansari, K. U., Gupta, N., and Bapat, S. K. (1994) An experimental and clinical evaluation of immunomodulating potential of human placental extracts. Indian J. Pharmacol. 26, 130-132.

3. Bohn, H. (1985) New pregnancy and placental proteins and their possible diagnostic significance. Behring Ins. Mitt. 78, 70-82.

4. Calleja-Agius, J., Muscat-Baron, Y., and Brincat, M. P. (2007) Skin ageing. Menopause Int. 13, 60-64.

5. Chakraborty, P. D. and Bhattacharyya, D. (2012) Aqueous extract of human placenta as a therapeutic agent. In: Recent Advances in Research on the Human Placenta. Zheng, J. (ed) InTech, Rijeka, Croatia, pp. 77-92.

6. Chakraborty, P. D., De, D., Bandyopadhyay, S., and Bhattacharyya, D. (2009) Human aqueous placental extract as a wound healer. J. Wound Care 18, 462, 464-467.

7. Corbel, M., Boichot, E., and Lagente, V. (2000) Role of gelatinases MMP-2 and MMP-9 in tissue remodeling following acute lung injury. Braz. J. Med. Biol. Res. 33, 749-754.

8. Cosgrove, M. C., Franco, O. H., Granger, S. P., Murray, P. G., and Mayes, A. E. (2007) Dietary nutrient intakes and skinaging appearance among middle-aged American women. Am. J. Clin. Nutr. 86, 1225-1131.

9. Darvin, M., Zastrow, L., Sterry, W., and Lademann, J. (2006) Effect of supplemented and topically applied antioxidant substances on human tissue. Skin Pharmacol. Physiol. 19, 238247.

10. Draelos, Z. D. (2010) Nutrition and enhancing youthful-appearing skin. Clinics Dermatol. 28, 400-408.

11. Gueniche, A., Benyacoub, J., Buetler, T. M., Smola, H., and Blum, S. (2006) Supplementation with oral probiotic bacteria maintains cutaneous immune homeostasis after UV exposure. Eur. J. Dermatol. 16, 511-517.

12. Han, N. R., Kim, K. Y., Kim, M. J., Kim, M. H., Kim, H. M., and Jeong, H. J. (2013) Porcine placenta mitigates proteinenergy malnutrition-induced fatigue. Nutrition 29, 1381-1387.

13. Koike, K., Yamamoto, Y., Suzuki, N., Yamazaki, R., Yoshikawa, C., Takuma, K., Sugiura, K., and Inoue, M. (2012) Efficacy of porcine placental extracts with hormone therapy for postmenopausal women with knee pain. Climacteric 15, 30-35.

14. Kong, C. S., Kim, J. A., Ahn, B., Byun, H. G., and Kim, S. K. (2010) Carboxymethylations of chitosan and chitin inhibit MMP expression and ROS scavenging in human fibrosarcoma cells. Process Biochem. 45, 179-186.

15. Liang, J., Pei, X., Zhang, Z., Wang, N., Wang, J., and Li, Y. (2010) The protective effects of long-term oral administration of marine collagen hydrolysate from chum salmon on collagen matrix homeostasis in the chronological aged skin of Sprague-Dawley male rats. J. Food Sci. 75, H230-238.

16. Madison, K. C. (2003) Barrier function of the skin: "la raison d'etre" of the epidermis. J. Invest. Dermatol. 121, 231-241.

17. Mitsui, Y., Bagchi, M., Marone, P. A., Moriyama, H., and Bagchi, D. (2015). Safety and toxicological evaluation of a 
novel, fermented, peptide-enriched, hydrolyzed swine placenta extract powder. Toxicol. Mech. Methods 25, 13-20.

18. Mittal, K. N. (1977) Role of placental extract in the therapy of urticarias. Indian J. Dermatol. 22, 117-120.

19. Morganti, P., Bruno, C., Guarneri, F., Cardillo, A., Del Ciotto, P., and Valenzano, F. (2002) Role of topical and nutritional supplement to modify the oxidative stress. Int. J. Cosmetic Sci. 24, 331-339.

20. Oh, J. H., Kim, Y. K., Jung, J. Y., Shin, J., Kim, K. H., Cho, K. H., Eun, H. C., and Chung J. H. (2011) Intrinsic aging- and photoaging-dependent level changes of glycosaminoglycans and their correlation with water content in human skin. $J$. Dermatol. Sci. 62, 192-201.

21. Onoue, S., Kobayashi, T., Takemoto, Y., Sasaki, I., and Shinkai, H. (2003) Induction of matrix metalloproteinase-9 secretion from human keratinocytes in culture by ultraviolet B irradiation. J. Dermatol. Sci. 33, 105-111.

22. Pal, P., Mallick, S., Mandal, S. K., Das, M., Dutta, A. K., Datta, P. K., Bera, R., and Bhadra, R. (2002) A human placental extract: in vivo and in vitro assessments of its melanocyte growth and pigment-inducing activities. Int. J. Dermatol. 41, 760-767.

23. Pal, P., Roy, R., Datta, P. K., Dutta, A. K., Biswas, B., and Bhadra, R. (1995) Hydroalcoholic human placental extract: skin pigmenting activity and gross chemical composition. Int. J. Dermatol. 34, 61-66.

24. Palombo, P., Fabrizi, G., Ruocco, V., Ruocco, E., Fluhr, J., Roberts, R., and Morganti, P. (2007) Beneficial long-term effects of combined oral/topical antioxidant treatment with the carotenoids lutein and zeaxanthin on human skin: a double-blind, placebo-controlled study. Skin Pharmacol. Phys. 20, 199-210.

25. Pinnell, S. R. (1982) Regulation of collagen synthesis. $J$. Inves. Dermatol. 79 Suppl 1, 73s-76s.

26. Schmittgen, T. D. and Livak, K. J. (2008) Analyzing realtime PCR data by the comparative C (T) method. Nat. Protoc. 3, 1101-1108.

27. Togashi, S., Takahashi, N., Iwama, M., Watanabe, S., Tamagawa, K., and Fukui, T. (2002) Antioxidative collagen-derived peptides in human-placenta extract. Placenta 23, 497502.

28. Tonello, G., Daglio, M., Zaccarelli, N., Sottofattori, E., Mazzei, M., and Balbi, A. (1996) Characterization and quantitation of the active polynucleotide fraction (PDRN) from human placenta, a tissue repair stimulating agent. J. Pharm. Biomed. Anal. 14, 1555-1560.

29. Wu, C. H., Chang, G. Y., Chang, W. C., Hsu, C. T., and Chen, R. S. (2003) Wound healing effects of porcine placental extracts on rats with thermal injury. Br. J. Dermatol. 148, 236245.

30. Yoshikawa, C., Takano, F., Ishigaki, Y., Okada, M., and Kyo, S. (2013) Effect of porcine placental extract on collagen production in human skin fibroblasts. Gynecol. Obstet. 3, 1-4.

31. Zague, V. (2008) A new view concerning the effects of collagen hydrolysate intake on skin properties. Arch. Dermatol. Res. 300, 479-483. 